\title{
Empathy, Mentalization, and Theory of Mind in Borderline Personality Disorder: Possible Overlap With Autism Spectrum Disorders
}

\author{
Nicoletta Vegni ${ }^{1 *}$, Caterina D'Ardia $^{2}$ and Giulia Torregiani ${ }^{1}$ \\ ${ }^{1}$ Faculty of Psychology, Niccolò Cusano University, Rome, Italy, ${ }^{2}$ Niccolò Cusano University, Rome, Italy
}

Keywords: theory of mind, empathy, borderline personality disorder, autism spectrum disorder, mentalization

\section{INTRODUCTION}

Autism spectrum disorder (ASD) is characterized by persistent deficits in social communication and interaction, behavior patterns, and narrow and repetitive interests or activities. Individuals with an ASD diagnosis have an atypical social approach to conversation reciprocity and a reduced sharing of interests, emotions, or feelings. Their verbal and non-verbal communication is poorly integrated, anomalies in eye contact and body language are present, as well as difficulties in understanding and using gestures (American Psychiatric Association, 2013). Over time the hypothesis has been confirmed that at the basis of the social compromises characterizing autism there are impairments in theory of mind (ToM), i.e., the ability to attribute mental states to people in order to explain and predict their behavior (Baron-Cohen et al., 1985; Baron-Cohen, 1995).

Some studies have related ASD to personality disorders, as shown by recent reviews of the

Edited by:

Drozdstoy Stoyanov Stoyanov, Plovdiv Medical University, Bulgaria

Reviewed by:

Vera Flasbeck

Ruhr University Bochum, Germany

Phil Reed,

Swansea University, United Kingdom

*Correspondence:

Nicoletta Vegni

nicoletta.vegni@unicusano.it

Specialty section:

This article was submitted to

Psychopathology,

a section of the journal

Frontiers in Psychology

Received: 05 November 2020

Accepted: 15 January 2021

Published: 11 February 2021

Citation:

Vegni N, D'Ardia C and Torregiani G

(2021) Empathy, Mentalization, and

Theory of Mind in Borderline

Personality Disorder: Possible Overlap

With Autism Spectrum Disorders.

Front. Psychol. 12:626353.

doi: 10.3389/fpsyg.2021.626353 psychopathological traits (Matson and Nebel-Schwalm, 2007; Matson and Goldin, 2013; Mannion et al., 2014). In relation to the analyses found in the literature, the disorder that in our opinion shows particular possible overlaps with ASD in terms of lack of empathy and theory of mind seems to be BPD.

Borderline personality disorder (BPD) is characterized by pervasive disadaptive modes of thought and behavior. BPD, as stated in DSM-5 (American Psychiatric Association, 2013), presents a pervasive pattern of interpersonal relationships, self-image and mood instability, and a marked impulsiveness, which generally begins in early adulthood in different contexts of daily life. Its distinctive features include emotional dysregulation, fear of neglect, malfunctioning in interpersonal relationships, fractioned thinking, and difficulty in impulse control.

Many studies have been conducted to identify the components of borderline personality disorder, including emotional dysregulation (Yen et al., 2002; Conklin et al., 2006), attachment (Fonagy, 2000), and theory of mind (Harari et al., 2010; Franzen et al., 2011). Emotional dysregulation is known as one of the main symptoms in patients with borderline personality disorder. Many studies have found that many borderline personality traits are the result of emotional dysregulation (Barnow et al., 2012; Ghiasi et al., 2016; Salgado et al., 2020). Studies have also revealed that people with this disorder have problems identifying, distinguishing, and integrating their own emotions with those of other individuals (Harari et al., 2010).

Recently, many have begun to consider empathy as a construct composed by two components, one cognitive and one affective (Baron-Cohen and Wheelwright, 2004; Shamay-Tsoory, 2011). Affective empathy involves the experience of the feelings and emotions of others through recognition, sensitivity to the emotions of others, and sharing the emotional experiences of others through an affective response appropriate to the situation of the other (Batchelder et al., 2017).

Cognitive empathy involves the process of understanding another person's perspective by adopting another person's point of view. The ability to adopt another person's point of view is 
consistent with the concept of theory of mind (ToM). Cognitive empathy also includes the ability to judge and understand the intentions of others in order to monitor one's own intentions (Batchelder et al., 2017).

This paper presents some opinions drawn from the analysis of literature from the last 20 years which has analyzed the link between ASD and personality disorders, and specifically BPD. The articles were selected according to the time criterion of the year of publication through the PUBMED database.

Papers that included the following keywords were considered: "borderline personality disorders and empathy,". "borderline personality disorders and theory of mind," "borderline personality disorders and autism," "autism and empathy," and "autism and theory of mind." Papers published between 2000 and 2020 were included.

The only articles outside the timeframe that were included were related to theoretical constructs.

\section{OVERLAPS BETWEEN BPD AND ASD}

Difficulties related to the social and relational fields are characteristics found in patients with BPD, as well as in patients with ASD. Considering patients with high functioning autism, Hofvander et al. (2009) showed that $68 \%$ of the sample, composed of adults with ASD, met the criteria for at least one personality disorder. In accordance with this, when checking for possible personality disorders in a group of young adults with Asperger's syndrome, a study showed a considerable overlap of symptoms between Asperger's syndrome and some personality disorders (Lugnegård et al., 2012). Baron-Cohen et al. (1985) emphasizes how the social and communicative difficulties of ASD subjects can be traced back to a deficiency of maturation of the theory of mind (ToM), or rather, of the cognitive mechanism responsible for the analysis of one's own and others' mental states, as well as highlighting in these subjects important difficulties in terms of empathy. Considering empathy as the ability to understand another's state of mind and systematization as the ability to perceive the models of change (the rules) that allow us to understand how things work and predict their future, some authors (Wheelwright et al., 2006) have developed a theory according to which different brain types are attributed to individuals according to their tendency to be more empathic or systematic.

Baron Cohen compared ASD with BPD in terms of lack of empathy and specifically with a "zero degree of empathy" measured with the Empathy Quotient (EQ) (Wheelwright et al., 2006; Baron-Cohen, 2011) as both disorders would manifest difficulties in social and interpersonal interactions as discriminating symptoms.

As for systematization ability, measured by the Systematization Quotient (SQ) (Goldenfeld et al., 2005; Wheelwright et al., 2006) patients with BPD would show low levels while patients with ASD would show higher levels than the average of normo-typical subjects. This difference would explain the tendency of ASD subjects to follow rules and the tendency to deviance and take risks in BPD subjects (Baron-Cohen, 2011). The study conducted by Dudas et al. (2017) analyzed a sample of subjects, including ASD, BPD, double-diagnosed, and normo-typical (NC) subjects compared according to $\mathrm{EQ}$ and SQ indices. In the SQ-R measure, all clinical groups had a statistically significant score that was higher than the control group. These data, which show high levels of systematization in patients with BPD as well as in patients with ASD, seem to contrast the idea that it is precisely systematization that differentiates BPD from ASD in relation to the tendency of the former to take risks and be impulsive and therefore to place themselves within a "spectrum of empathy" equal to negative zero rather than positive (Baron-Cohen, 2011).

It has been clarified in the literature that a lack of empathy is a hallmark of ASD (Harmsen, 2019; Stroth et al., 2019).

At the same time, scientific literature presents various evidence in favor of empathic and mentalization deficits in $\mathrm{BPD}$, which can be interpreted as possible causes of social and relational problems. Baron-Cohen describes the empathic difficulties of patients with BPD, in relation to the recognition and response components, describing them as deficient in reacting to others with an appropriate emotion (empathic response), and with difficulty in effectively and precisely determining the intentions and emotions of others' facial expressions (Baron-Cohen, 2011) In a study, how ambiguity of stimuli can lead to a reduction in cognitive or emotional empathy in BPD was discussed (Niedtfeld, 2017). Video clips were shown to 34 patients with BPD and 32 controls, through which vocal content, prosodies, and facial expressions were presented. BPD patients showed greater emotional empathy when the proposed stimuli included emotions expressed in a non-verbal way, while with regard to cognitive empathy, there were no significant differences between BPD and controls. These results suggest that subjects with BPD show altered emotional empathy, experiencing higher rates of emotional contagion when emotions are expressed non-verbally. The latter may contribute to misunderstandings and inappropriate social behavior. Differences in amygdala activation in subjects with BPD compared to control subjects were also found in a study revealing difficulty in understanding neutral facial expressions, often interpreting them as threatening (Donegan et al., 2003). Regarding the analysis of the different components of empathy involved in BPD, using a self-report measure of empathy, the Interpersonal Reactivity Index (IRI; Davis, 1983), a study showed that women diagnosed with BPD showed higher average levels of affective empathy and lower average levels of cognitive empathy, compared to a control sample of women with anorexia nervosa and an undiagnosed control group (Guttman and Laporte, 2002). These results were confirmed by another research that demonstrated a "double dissociation" of cognitive and affective empathy in BPD, suggesting that the behavioral difficulties manifested in BPD can be explained by a dysfunctional model of empathic capacity (Harari et al., 2010). The authors started from the hypothesis that the interpersonal malfunction typical of BPD would find justification in their low levels of cognitive empathy and theory of mind, while higher levels of affective empathy would account for emotional hyperactivity.

To evaluate the cognitive and affective aspects of empathy IRI was used, while the faux pas recognition test was adopted for measurements related to the theory of mind (Phillips et al., 1998). The results found that the controls had higher IRI scores 
in the cognitive component of empathy than in the affective component, whereas the BPD group showed the opposite pattern. Significant differences were shown between BPD patients and the control group in the understanding of the theory of the cognitive mind but not in the emotional one, detected with the faux pas recognition test. In the study BPD patients showed worse performance in both cognitive empathy and cognitive mind theory measures, but there were no differences in the understanding of emotional mind theory, while the affective aspects of empathy were even better in patients with BPD. Providing support in terms of neurobiological comparison to the "dissociation" of cognitive and affective empathy in BPD, a study, including fMRI data, showed that patients with BPD compared to controls showed lower activation during cognitive empathy activities in the upper temporal fissure (STS), an area associated with the inference of other people's mental states (Zaki et al., 2009), but greater activation in the medium insular cortex (Jackson et al., 2006), an area associated with personal distress, during activities involving emotional empathy (Dziobek et al., 2011). Moreover, considering the typical manifestations of the borderline personality, two processes were put in relation to empathic dysfunctions: emotional dysregulation, i.e., highly uncontrolled emotional reactivity, an inability to modulate internal emotions, resulting in an inappropriate emotional and behavioral response (Linehan, 1993; Gratz et al., 2006) and hypermentalization, i.e., a misinterpretation or a sort of super reference of one's own and others' thoughts and feelings (Sharp et al., 2011, 2013). Another study examined the relationships between emotional dysregulation, hyper-mentalization, and cognitive and affective empathy in 252 adolescent patients, divided into BPD subjects and undiagnosed subjects, revealing that in both groups, emotional dysregulation was related to increased affective empathy. Hyper-mentalization, on the other hand, was correlated with reduced cognitive empathy in patients with BPD, while hyper-mentalization was not correlated with any kind of empathy in patients without BPD (Kalpakci et al., 2016). As regards, difficulties in mentalization or the ability to precisely infer the mental states of others, a dysfunctional mechanism of these abilities was hypothesized at the basis of borderline personality disorder (Fonagy et al., 1991; Sharp and Fonagy, 2008). Moreover, with the aim of examining the mediating role of the regulation of emotions in the relationship between ToM and borderline traits in adolescents, a study found a trend that associates an overall reduced ToM capacity with an increase in borderline traits and a consistent correlation between these and difficulties in emotional regulation; not an absence of mentalization capacity but an over-mentalization (Sharp et al., 2011). This hyper-mentalization would manifest itself as an excessive interpretation of the mental state of others. The tendency to make overly complex inferences about social signals that have been found to be erroneous has also been shown. These subjects would tend to over-interpret social signals by overestimating them. These results suggest that the

\section{REFERENCES}

American Psychiatric Association. (2013). Diagnostic and Statistical Manual of Mental Disorders (Fifth Edition). Arlington, VA: American Psychiatric Association. doi: 10.1176/appi.books.9780890425596 difficulties of emotional dysregulation, at least in part, mediate the association between hyper-mentalization and BPD.

\section{DISCUSSION}

It should be clarified that there are often overlaps in literature between the different cognitive and affective components of empathy and mentalization, and there is therefore no unanimous consensus on this issue. Moreover, some authors understand the theory of mind in terms of the "presence or absence" of this ability with the consequent effective functioning or not of the psychic activities linked to the attribution of mental states; others consider it as part of a wider mental activity, i.e., metacognition, which would include, in addition to ToM, more sophisticated mentalization abilities. It seems, therefore, that common characteristics of serious personality disorders can be traced back to alterations in metathought and emotional regulation, both of which in turn are expressions of metacognitive functions. Each particular disorder is characterized by a specific profile of impairment (more or less serious) of some subfunctions of metacognition. In particular, the borderline disorder as opposed to autism would be characterized by a difficulty to distinguish between representation and reality, to modulate one's mental states functionally and adaptively, and to integrate the various elements of mental activity (sensations, emotions, thoughts, etc.) into continuous and coherent narrations of oneself, others, and the world.

The studies presented have uniformly shown that the lack of empathy and theory of mind is an overlapping aspect in BPD and ASD but there is still little evidence regarding the differences between the two clinical domains with respect to the affective and cognitive components of empathy itself. Furthermore, in the studies, analyzed measurements are carried out with different instruments and tests, which means that the results of one study cannot be fully compared with those of another.

It would be useful, also with regard to the development of possible integrated therapies, to investigate the link between BPD and ASD in terms of lack of empathy, ToM, and mentalization using shared protocols for diagnosis that can allow homogeneous measurements that do not leave room for the interpretation of individual scores.

Finally, it would be interesting to investigate possible overlapping between BPD and ASD with respect to executive functions and impulse control, an aspect not yet particularly stressed in the scientific literature.

\section{AUTHOR CONTRIBUTIONS}

NV: developed the topic of the work and carried out the final linguistic review. CD'A: contribution for the ASD topic. GT: references research. All authors contributed to the article and approved the submitted version.

Barnow, S., Limberg, A., Stopsack, M., Spitzer, C., Grabe, H. J., Freyberger, H. J., et al. (2012). Dissociation and emotion regulation in borderline personality disorder. Psychol. Med. 42, 783-794. doi: 10.1017/S0033291711001917

Baron-Cohen, S. (1995). Mindblindness: An Essay on Autism and Theory of Mind. (pp. xxii, 171). Boston, MA: The MIT Press. 
Baron-Cohen, S. (2011). The Science of Evil: On Empathy and the Origins of Cruelty. (pp. xii, 240). New York, NY: Basic Books.

Baron-Cohen, S., Leslie, A. M., and Frith, U. (1985). Does the autistic child have a "theory of mind?" Cognition 21, 37-46. doi: 10.1016/0010-0277(85)90022-8

Baron-Cohen, S., and Wheelwright, S. (2004). The empathy quotient: an investigation of adults with asperger syndrome or high functioning autism, and normal sex differences. J. Autism Dev. Disord. 34, 163-175. doi: 10.1023/B:JADD.0000022607.19833.00

Batchelder, L., Brosnan, M., and Ashwin, C. (2017). The development and validation of the empathy components questionnaire (ECQ). PLoS ONE 12:e0169185. doi: 10.1371/journal.pone.0169185

Conklin, C. Z., Bradley, R., and Westen, D. (2006). Affect regulation in borderline personality disorder. J. Nerv. Ment. Dis. 194, 69-77. doi: 10.1097/01.nmd.0000198138.41709.4f

Davis, M. H. (1983). The effects of dispositional empathy on emotional reactions and helping: a multidimensional approach. J. Pers. 51, 167-184. doi: 10.1111/j.1467-6494.1983.tb00860.x

Donegan, N. H., Sanislow, C. A., Blumberg, H. P., Fulbright, R. K., Lacadie, C., Skudlarski, P., et al. (2003). Amygdala hyperreactivity in borderline personality disorder: implications for emotional dysregulation. Biol. Psychiatry 54, 1284-1293. doi: 10.1016/S0006-3223(03)00636-X

Dudas, R. B., Lovejoy, C., Cassidy, S., Allison, C., Smith, P., and Baron-Cohen, S. (2017). The overlap between autistic spectrum conditions and borderline personality disorder. PLOS ONE 12:e0184447. doi: 10.1371/journal.pone. 0184447

Dziobek, I., Preißler, S., Grozdanovic, Z., Heuser, I., Heekeren, H. R., and Roepke, S. (2011). Neuronal correlates of altered empathy and social cognition in borderline personality disorder. Neuroimage 57, 539-548. doi: 10.1016/j.neuroimage.2011.05.005

Fonagy, P. (2000). Attachment and borderline personality disorder. J. Am. Psychoanal Assoc. 48, 1129-1146; discussion 1175-87. doi: $10.1177 / 00030651000480040701$

Fonagy, P., Steele, H., and Steele, M. (1991). Maternal representations of attachment during pregnancy predict the organization of infant-mother attachment at one year of age. Child Dev. 62:891. doi: 10.2307/1131141

Franzen, N., Hagenhoff, M., Baer, N., Schmidt, A., Mier, D., Sammer, G., et al. (2011). Superior 'theory of mind' in borderline personality disorder: an analysis of interaction behavior in a virtual trust game. Psychiatry Res. 187, 224-233. doi: 10.1016/j.psychres.2010.11.012

Ghiasi, H., Mohammadi, A., and Zarrinfar, P. (2016). An investigation into the roles of theory of mind, emotion regulation, and attachment styles in predicting the traits of borderline personality disorder. Iran. J. Psychiatry 11, 206-213.

Goldenfeld, N., Baron-Cohen, S., and Wheelwright, S. (2005). Empathizing and systemizing in males, females, and autism. Clin. Neuropsych. 2, 338-345.

Gratz, K. L., Rosenthal, M. Z., Tull, M. T., Lejuez, C. W., and Gunderson, J. G. (2006). An experimental investigation of emotion dysregulation in borderline personality disorder. J. Abnorm. Psychol. 115, 850-855. doi: 10.1037/0021-843X.115.4.850

Guttman, H., and Laporte, L. (2002). Alexithymia, empathy, and psychological symptoms in a family context. Compr. Psychiatry 43, 448-455. doi: 10.1053/comp.2002.35905

Harari, H., Shamay-Tsoory, S. G., Ravid, M., and Levkovitz, Y. (2010). Double dissociation between cognitive and affective empathy in borderline personality disorder. Psychiatry Res. 175, 277-279. doi: 10.1016/j.psychres.2009.03.002

Harmsen, I. E. (2019). Empathy in autism spectrum disorder. J. Autism Dev. Disord. 49, 3939-3955. doi: 10.1007/s10803-019-04087-w

Hofvander, B., Delorme, R., Chaste, P., Nydén, A., Wentz, E., Ståhlberg, O., et al. (2009). Psychiatric and psychosocial problems in adults with normal-intelligence autism spectrum disorders. BMC Psychiatry 9:35. doi: 10.1186/1471-244X-9-35

Jackson, P. L., Brunet, E., Meltzoff, A. N., and Decety, J. (2006). Empathy examined through the neural mechanisms involved in imagining how I feel versus how you feel pain. Neuropsychologia 44, 752-761. doi: 10.1016/j.neuropsychologia.2005.07.015

Kalpakci, A., Vanwoerden, S., Elhai, J. D., and Sharp, C. (2016). The Independent Contributions of Emotion Dysregulation and Hypermentalization to the "Double Dissociation" of Affective and Cognitive Empathy in
Female Adolescent Inpatients With BPD. J. Pers. Disord. 30, 242-260. doi: 10.1521/pedi_2015_29_192

Linehan, M. (1993). Cognitive-Behavioral Treatment of Borderline Personality Disorder. Guilford Press.

Lugnegård, T., Hallerbäck, M. U., and Gillberg, C. (2012). Personality disorders and autism spectrum disorders: what are the connections? Compr. Psychiatry 53, 333-340. doi: 10.1016/j.comppsych.2011.05.014

Mannion, A., Brahm, M., and Leader, G. (2014). Comorbid psychopathology in autism spectrum disorder. Rev. J. Autism Dev. Disorders 1, 124-134. doi: 10.1007/s40489-014-0012-y

Matson, J. L., and Goldin, R. L. (2013). Comorbidity and autism: trends, topics and future directions. Res. Autism Spectr. Disord. 7, 1228-1233. doi: 10.1016/j.rasd.2013.07.003

Matson, J. L., and Nebel-Schwalm, M. S. (2007). Comorbid psychopathology with autism spectrum disorder in children: an overview. Res. Dev. Disabil. 28, 341-352. doi: 10.1016/j.ridd.2005.12.004

Niedtfeld, I. (2017). Experimental investigation of cognitive and affective empathy in borderline personality disorder: effects of ambiguity in multimodal social information processing. Psychiatry Res. 253, 58-63. doi: 10.1016/j.psychres.2017.03.037

Phillips, W., Baron-Cohen, S., and Rutter, M. (1998). Understanding intention in normal development and in autism. Br. J. Dev. Psychol. 16, 337-348. doi: 10.1111/j.2044-835X.1998.tb00756.x

Salgado, R. M., Pedrosa, R., and Bastos-Leite, A. J. (2020). Dysfunction of empathy and related processes in borderline personality disorder: a systematic review. Harv. Rev. Psychiatry 28, 238-254. doi: 10.1097/HRP.0000000000000260

Shamay-Tsoory, S. G. (2011). The neural bases for empathy. Neuroscientist 17, 18-24. doi: $10.1177 / 1073858410379268$

Sharp, C., and Fonagy, P. (2008). "Social cognition and attachmentrelated disorders," in Social Cognition and Developmental Psychopathology (New York, NY: Oxford University Press), 271-302. doi: 10.1093/med/9780198569183.003.0010

Sharp, C., Ha, C., Carbone, C., Kim, S., Perry, K., Williams, L., et al. (2013). Hypermentalizing in adolescent inpatients: treatment effects and association with borderline traits. J. Pers. Disord. 27, 3-18. doi: 10.1521/pedi.2013.27.1.3

Sharp, C., Pane, H., Ha, C., Venta, A., Patel, A. B., Sturek, J., et al. (2011). Theory of mind and emotion regulation difficulties in adolescents with borderline traits. J. Am. Acad. Child Adolesc. Psychiatry,50, 563-573.e1. doi: 10.1016/j.jaac.2011.01.017

Stroth, S., Paye, L., Kamp-Becker, I., Wermter, A.-K., Krach, S., Paulus, F. M., et al. (2019). Empathy in females with autism spectrum disorder. Front. Psychiatry 10:428. doi: $10.3389 /$ fpsyt.2019.00428

Wheelwright, S., Baron-Cohen, S., Goldenfeld, N., Delaney, J., Fine, D., Smith, R., et al. (2006). Predicting autism spectrum quotient (AQ) from the systemizing quotient-revised (SQ-R) and empathy quotient (EQ). Brain Res. 1079, 47-56. doi: 10.1016/j.brainres.2006.01.012

Yen, S., Shea, M. T., Battle, C. L., Johnson, D. M., Zlotnick, C., DolanSewell, R., et al. (2002). Traumatic exposure and posttraumatic stress disorder in borderline, schizotypal, avoidant, and obsessivecompulsive personality disorders: findings from the collaborative longitudinal personality disorders study. J. Nerv. Ment. Dis. 190, 510-518. doi: 10.1097/00005053-200208000-00003

Zaki, J., Weber, J., Bolger, N., and Ochsner, K. (2009). The neural bases of empathic accuracy. Proc. Natl. Acad. Sci. U.S.A. 106, 11382-11387. doi: $10.1073 /$ pnas.0902666106

Conflict of Interest: The authors declare that the research was conducted in the absence of any commercial or financial relationships that could be construed as a potential conflict of interest.

Copyright (๑ 2021 Vegni, D’Ardia and Torregiani. This is an open-access article distributed under the terms of the Creative Commons Attribution License (CC BY). The use, distribution or reproduction in other forums is permitted, provided the original author(s) and the copyright owner(s) are credited and that the original publication in this journal is cited, in accordance with accepted academic practice. No use, distribution or reproduction is permitted which does not comply with these terms. 